\title{
REFLECTIONLESS POTENTIALS FOR AN ORDINARY DIFFERENTIAL OPERATOR OF ORDER FOUR
}

\author{
A. LAPTEV, R. SHTERENBERG, V. SUKHANOV AND J. ÖSTENSSON
}

\begin{abstract}
The aim of this paper is to construct exact formulae for reflectionless potentials for ordinary differential operators of order four. They lead to soliton type solutions which are well known for one dimensional Schrödinger operators. Such solitons are solutions of some non-linear integrable systems appeared in [8] (see also [9]).
\end{abstract}

\section{INTRODUCTION}

Let us consider a fourth order selfadjoint differential operator

$$
L=\frac{d^{4}}{d x^{4}}+\frac{d}{d x} u(x) \frac{d}{d x}+v(x) .
$$

We assume that potentials $u(x)$ and $v(x)$ are real-valued, smooth and rapidly decaying functions satisfying the property

$$
|u(x)|,|v(x)|<\text { const } \exp (-\varepsilon|x|)
$$

for some $\varepsilon>0$.

This article could be considered as a natural continuation of the study started in [9] (see also [13], where some of the result of this paper were presented). Namely we shall give a full description of potentials $u$ and $v$ which the operator $L$ is reflection free. Naturally such a class of potentials is related to the discrete spectrum which in the case of fourth order operators can be either negative or embedded into the continuous spectrum. However, we find at least two following surprising facts. We prove that if a reflectionless operator $L$ has a negative eigenvalue, then this eigenvalue has to be of multiplicity two. The second fact concerns reflectionless potentials which create a positive eigenvalue embedded into the continuous spectrum. In this case we prove that the operator $L$ is equal to square of a Schrödinger operator with the soliton type potential $\cosh ^{-2}$. Of course such an eigenvalue is of multiplicity one. In the end we also include so-called non-spectral singularities and obtain a non-trivial class of reflectionless operators which does not generate any eigenvalue.

1991 Mathematics Subject Classification. Primary 35P15; Secondary 35L15, 47A75, $35 \mathrm{~J} 10$. 
Compare with [9] where most of the main results were obtained by guessing and using complicated computations applying Darboux transforms, in this paper we are able to present full description of reflectionless potentials by systematically studying a Riemann-Hilbert problem. Such an approach has shown to be very fruitful in the study of higher order operators [1], [10], where soliton type solutions were not considered. Notice that the RiemannHilbert approach was also used in a number of related papers [2], [4], [5], [6], [11] and [12].

Considering non-spectral singularities we are able to clarify the recent result from [3], where the authors constructed an example violating a functional inequality related to a trace formula for the operator (1.1) (see Remark 3, Section 6).

\section{RIEMANN-HILBERT PROBLEM}

2.1. Reduction to an integral equation. Here we would like to remind a formulation of the Riemann-Hilbert problem adapted to a fourth order differential operator (see [1] and [10]).

Let us introduce the following four solutions $\Psi_{l}(x, k), l=1,2,3,4$, of the spectral equation

$$
L \Psi=\frac{d^{4}}{d x^{4}} \Psi+\frac{d}{d x} u \frac{d}{d x} \Psi+v \Psi=k^{4} \Psi
$$

such that

$$
\Psi_{l}(x, k)=e^{k_{l} x}(1+o(1)), x \rightarrow+\infty
$$

and

$$
\Psi_{l}(x, k)=e^{k_{l} x} O(1), x \rightarrow-\infty,
$$

where $k_{l}=k \exp (i \pi(l-1) / 2), l=1,2,3,4$. Notice that $k_{l}=k_{l}(k)$, $k \in \mathbb{C}$. By using standard computations one can find that $\Psi_{l}(x, k)$ satisfy the following Fredholm integral equations

$$
\begin{aligned}
& \frac{1}{4 k^{3}} \int_{x}^{+\infty} \sum_{j: \operatorname{Re}\left(k_{j}\right) \geq \operatorname{Re}\left(k_{l}\right)} e^{k_{j}(x-y)}\left(\left(k_{j}\right)^{2} u(y)+k_{j} u^{\prime}(y)+v(y)\right) \Psi_{l}(y, k) d y \\
& -\frac{1}{4 k^{3}} \int_{-\infty}^{x} \sum_{j: \operatorname{Re}\left(k_{j}\right)<\operatorname{Re}\left(k_{l}\right)} e^{k_{j}(x-y)}\left(\left(k_{j}\right)^{2} u(y)+k_{j} u^{\prime}(y)+v(y)\right) \Psi_{l}(y, k) d y .
\end{aligned}
$$

Such solutions are meromorphic functions in sectors $\Omega_{m}, m=1, \ldots 8$,

$$
\Omega_{m}=\{k: \pi(m-1) / 4<\arg k<\pi m / 4\}
$$


and have jumps on the set of rays $\gamma_{m}$

$$
\gamma_{m}=\{k: \arg k=m \pi / 4\} .
$$

It is well known that functions $\Psi_{l}(x, k)$ might have singularities. Some of them correspond to the spectrum of the operator $L$ and therefore lie on the rays (2.5). However, there are poles which are usually called non-spectral singularities and do not belong $\gamma_{m}$. Both such singularities appear from the fact that the equations (2.4) are of Fredholm type. Due to the condition (1.2) the solutions $\Psi_{l}(x, k)$ have analytic continuation to some neibourhoods $\Omega_{m}^{\varepsilon}$ of sectors $\Omega_{m}$, where

$$
\Omega_{m}^{\varepsilon}=\{k:(m-1) \pi / 4-\varepsilon<\arg k<m \pi / 4+\varepsilon\} .
$$

In particular, this means that the singularities of $\Psi_{l}(x, k)$ cannot have accumulating points belonging to the rays (2.5). From the equation (2.4) one immediately derives that functions $\Psi_{l}(x, k)$ satisfy the following asymptotics as $|k| \rightarrow \infty$ uniformly with respect to $x$ :

$$
\Psi_{l}(x, k)=e^{k_{l} x}(1+o(1)) .
$$

This allows us to conclude that the set of singularities of functions $\left\{\Psi_{l}(x, k)\right\}_{l=1}^{4}$ is finite.

Let us now consider asymptotics of the functions $\Psi_{l}(x, k)$, as $x \rightarrow-\infty$, and introduce

$$
a_{l}(k)=1+\frac{1}{4 k^{3}} \int_{-\infty}^{+\infty} e^{-k_{l} y}\left(\left(k_{l}\right)^{2} u(y)+k_{l} u^{\prime}(y)+v(y)\right) \Psi_{l}(y, k) d y .
$$

Then the asymptotics (2.3) can be written in a more transparent form

$$
\Psi_{l}(x, k)=e^{k_{l} x}\left(a_{l}(k)+o(1)\right), \quad x \rightarrow-\infty, \quad k \in \cup_{m=1}^{8} \Omega_{m} .
$$

Functions $a_{l}(k)$ have the same analytical properties as the functions $\Psi_{l}(x, k)$. In particular, they are meromorphic in sectors $\Omega_{m}$.

2.2. Some algebraic properties of $\Psi_{1}(\mathbf{x}, \mathbf{k})$. Let us introduce a Wronskian of three functions

$$
\mathcal{W}_{3}[f, g, h]=\left|\begin{array}{ccc}
f & g & h \\
f^{\prime} & g^{\prime} & h^{\prime} \\
f^{\prime \prime} & g^{\prime \prime} & h^{\prime \prime}
\end{array}\right|
$$

Lemma 2.1. If $f(x, k), g(x, k), h(x, k)$ are three solutions of the spectral equation (2.1), then so is the following Wronskian

$$
\overline{\mathcal{W}_{3}[f(x,-\bar{k}), g(x,-\bar{k}), h(x,-\bar{k})] .}
$$

This is a well-known statement which follows from Liouville's formula. 
Lemma 2.2. Let $L$ be the operator defined in (1.1) with real potentials $u$ and $v$. Then solutions $\Psi_{l}(x, k)$ defined by the boundary conditions (2.2) and (2.3) satisfy the following relations:

$$
\begin{gathered}
\Psi_{1}(x, k)=\overline{\Psi_{1}(x, \bar{k})}, \quad \Psi_{3}(x, k)=\overline{\Psi_{3}(x, \bar{k})}, \\
\Psi_{2}(x, k)=\overline{\Psi_{4}(x, \bar{k})}, \quad \Psi_{1}(x, k)=\overline{\Psi_{4}(x, i \bar{k})}, \quad \Psi_{2}(x, k)=\overline{\Psi_{3}(x, i \bar{k}) .}
\end{gathered}
$$

Proof. The left hand sides of all these relations are solutions of the equation (2.1) and satisfy the same conditions at $\pm \infty$ as the functions at the right hand side. This implies that they must be equal.

By using Lemma 2.2 and taking into account (2.2) and (2.8) we obtain the following statement:

Lemma 2.3. Let $a_{l}(k)$ be the functions defined in (2.7). Then

$$
\begin{gathered}
a_{1}(k)=\overline{a_{1}(\bar{k})}, \quad a_{3}(k)=\overline{a_{3}(\bar{k})}, \\
a_{2}(k)=\overline{a_{4}(\bar{k})}, \quad a_{1}(k)=\overline{a_{4}(i \bar{k})}, \quad a_{2}(k)=\overline{a_{3}(i \bar{k})} .
\end{gathered}
$$

Further interesting relations between the coefficients $a_{l}$ were first found in [13].

Lemma 2.4. The functions $a_{l}(k)$ satisfy the identities

$$
a_{1}(k) a_{3}(k)=1, \quad a_{2}(k) a_{4}(k)=1 .
$$

Proof. The proofs of the two identities (2.9) are similar, so we consider only the first one. Let us now introduce a Wronskian of two functions

$$
\mathcal{W}_{2}[f, g]=f^{\prime \prime \prime} g^{\prime}-f^{\prime \prime} g^{\prime}+f^{\prime} g^{\prime \prime}-f g^{\prime \prime \prime} .
$$

Using (2.1) we obtain

$$
\left(\mathcal{W}_{2}\left[\Psi_{1}, \Psi_{3}\right]\right)^{\prime}=\Psi_{1}^{\prime \prime \prime \prime} \Psi_{3}-\Psi_{1} \Psi_{3}^{\prime \prime \prime \prime}=\left(u \Psi_{1} \Psi_{3}^{\prime}-u \Psi_{1}^{\prime} \Psi_{3}\right)^{\prime}
$$

and therefore

$$
\mathcal{W}_{2}\left[\Psi_{1}, \Psi_{3}\right](c)-\mathcal{W}_{2}\left[\Psi_{1}, \Psi_{3}\right](-c)=\left.\left(u \Psi_{1} \Psi_{3}^{\prime}-u \Psi_{1}^{\prime} \Psi_{3}\right)\right|_{-c} ^{c}
$$

Due to (1.2), (2.2) and (2.3) the limit of right hand side of this equality equals zero as $c \rightarrow+\infty$. Thus

$$
\lim _{x \rightarrow-\infty} \mathcal{W}_{2}\left[\Psi_{1}, \Psi_{3}\right](x, k)=\lim _{x \rightarrow+\infty} \mathcal{W}_{2}\left[\Psi_{1}, \Psi_{3}\right](x, k)
$$

The latter equality together with asymptotics (2.2) and (2.8) (which hold true even together with the derivatives with respect to $x$ ) completes the proof of the lemma. 
Let $\Psi:=\left(\Psi_{1}, \Psi_{2}, \Psi_{3}, \Psi_{4}\right)^{t}$. Denote by $\Psi^{+m}\left(\Psi^{-m}\right)$ the limits of $\Psi$ as $k$ approaches the ray $\gamma_{m}$ such that $\arg k \rightarrow \pi m / 4 \pm 0$. Let $G_{m}(k)$ be matrices which connect vector-functions $\Psi^{+m}(x, k)$ and $\Psi^{-m}(x, k)$

$$
\Psi^{+m}(x, k)=G_{m}(k) \Psi^{-m}(x, k) .
$$

If $m=0$, then

$$
\Psi^{(+0)}(x, k)=\left(\begin{array}{c}
e^{k x} \\
e^{i k x} \\
e^{-k x} \\
e^{-i k x}+r_{0}(k) e^{i k x}
\end{array}\right)(1+o(1)), \quad x \rightarrow+\infty
$$

respectively

$$
\Psi^{(-0)}(x, k)=\left(\begin{array}{c}
e^{i k x}+\frac{e^{k x}}{r_{0}(k)} e^{-i k x} \\
e^{-k x} \\
e^{-i k x}
\end{array}\right)(1+o(1)), \quad x \rightarrow+\infty
$$

where $r_{0}$ can be found from (2.4) as

$$
r_{0}(k)=\frac{1}{4 k^{3}} \int_{-\infty}^{\infty} e^{k_{2} y}\left(\left(k_{2}\right)^{2} u(y)+k_{2} u^{\prime}(y)+v(y)\right) \Psi_{4}(y, k+i 0) d y .
$$

Therefore

If $m=1$, then

$$
G_{0}(k)=\left(\begin{array}{cccc}
1 & 0 & 0 & 0 \\
0 & 1 & 0 & -\bar{r}_{0} \\
0 & 0 & 1 & 0 \\
0 & r_{0} & 0 & 1-\left|r_{0}\right|^{2}
\end{array}\right)
$$

$$
\Psi^{(+1)}(x, k)=\left(\begin{array}{c}
e^{k x} \\
e^{i k x} \\
e^{-k x}+r_{1}(k) e^{i k x} \\
e^{-i k x}+r_{2}(k) e^{k x}
\end{array}\right)(1+o(1)), \quad x \rightarrow+\infty
$$

and

$$
\Psi^{(-1)}(x, k)=\left(\begin{array}{c}
e^{k x}+\overline{r_{2}(k)} e^{-i k x} \\
e^{i k x}+\overline{r_{1}(k)} e^{-k x} \\
e^{-k x} \\
e^{-i k x}
\end{array}\right)(1+o(1)), \quad x \rightarrow+\infty
$$

where

$$
\begin{aligned}
& r_{1}(k)=\frac{1}{4 k^{3}} \int_{-\infty}^{\infty} e^{k_{2} y}\left(\left(k_{2}\right)^{2} u(y)+k_{2} u^{\prime}(y)+v(y)\right) \Psi_{3}\left(y, k e^{i 0}\right) d y \\
& r_{2}(k)=\frac{1}{4 k^{3}} \int_{-\infty}^{\infty} e^{k_{1} y}\left(\left(k_{1}\right)^{2} u(y)+k_{1} u^{\prime}(y)+v(y)\right) \Psi_{4}\left(y, k e^{i 0}\right) d y .
\end{aligned}
$$


Thus

$$
G_{1}(k)=\left(\begin{array}{cccc}
1 & 0 & 0 & -\bar{r}_{2} \\
0 & 1 & -\bar{r}_{1} & 0 \\
0 & r_{1} & 1-\left|r_{1}\right|^{2} & 0 \\
r_{2} & 0 & 0 & 1-\left|r_{2}\right|^{2}
\end{array}\right) .
$$

By using the invariance of the equation (2.1) under transformation $k \rightarrow i k$ one finds matrices $G_{3}$ and $G_{4}$

$$
G_{m+2}(i k)=\sigma G_{m}(k) \sigma^{-1}, \quad \sigma=\left(\begin{array}{cccc}
0 & 1 & 0 & 0 \\
0 & 0 & 1 & 0 \\
0 & 0 & 0 & 1 \\
1 & 0 & 0 & 0
\end{array}\right) .
$$

It is natural to call the functions $r_{l}(k), l=0,1,2$, the reflection coefficients.

\section{REFLECTIONLESS POTENTIALS GENERATED BY ONE NEGATIVE EIGENVALUE}

3.1. A negative eigenvalue is always of multiplicity two. We define reflectionless potentials as potentials for which all solutions $\Psi_{l}(x, k)$ are meromorphic in $k$ functions. For such potentials jump conditions at $\gamma_{m}$ are trivial and reflection coefficients are equal to zero

$$
r_{0}(k)=r_{1}(k)=r_{2}(k)=0 .
$$

Let us consider reflectionless case corresponding to one negative eigenvalue $\lambda_{*}=k_{*}^{4}, k_{*}=(1+i) s_{*}, s_{*}>0$, and such that the functions $\Psi_{l}(x, k)$ do not have any other spectral and non-spectral singularities.

The boundary conditions (2.2), (2.8) at $\pm \infty$ imply $a_{2}^{(-1)}\left(k_{*}\right)=0$ or $a_{3}^{(-1)}\left(k_{*}\right)=0$, where $a_{l}^{( \pm m)}$ are the limits of $a_{l}$ as $\arg k \rightarrow \pi m / 4 \pm 0$. On the other hand from the integral equations (2.4) one obtains that $\Psi_{2}^{(+1)}=\overline{\Psi_{3}^{(-1)}}$. Therefore by using (2.7) we find

$$
a_{2}^{(+1)}(k)=\overline{a_{3}^{(-1)}(k)} .
$$

Clearly $\Psi_{2}\left(x, k_{*}\right)$ and $\Psi_{3}\left(x, k_{*}\right)$ are exponentially decaying functions as $x \rightarrow+\infty$. It follows from (3.1) that the functions $a_{2}(k)$ and $a_{3}(k)$ are meromorphic in the first quadrant $\{k: 0<\arg k<\pi / 2\}$ and $k_{*}$ is their common zero. Thus (2.8) implies that $\Psi_{2}\left(x, k_{*}\right)$ and $\Psi_{3}\left(x, k_{*}\right)$ are two linear independent eigenfunctions of the operator $L$.

In particular, since the multiplicity of the negative eigenvalue of the operator $L$ cannot exceed two, this means that $k_{*}$ is a simple zero for each of the functions $a_{2}(k)$ and $a_{3}(k)$.

We finally obtain that for a reflectionless pair of potentials $u$ and $v$ the negative eigenvalue $\lambda_{*}$ of the operator $L$ is always of multiplicity two. 
3.2. Properties of eigenfuntions. Let us now consider meromorphic solutions $\Psi_{l}(x, k)$ at $k_{*}$. If $k \in \gamma_{1}$ then for the functions $\Psi_{2}(x, k)$ and $\Psi_{3}(x, k)$ the sum in the second integral in (2.4) disappears and therefore these equations become Volterra's integral equations. This fact implies that both $\Psi_{2}(x, k)$ and $\Psi_{3}(x, k)$ are analytic at $k_{*}$. Since $k_{*}$ is a simple zero of functions $a_{2}(k)$ and $a_{3}(k)$, in view of Lemma 2.4 we find that $a_{1}(k)$ and $a_{4}(k)$ have simple poles of multiplicity one at $k_{*}$.

Let $\tilde{\Psi}_{l}(x, k)$ be solutions of our differential equation (2.1) satisfying the following boundary conditions at $\pm \infty$ :

$$
\begin{gathered}
\tilde{\Psi}_{l}(x, k)=e^{k_{l} x}(1+o(1)), \quad x \rightarrow-\infty, \\
\tilde{\Psi}_{l}(x, k)=e^{k_{l} x} O(1), \quad x \rightarrow+\infty .
\end{gathered}
$$

Then

$$
\begin{gathered}
\tilde{\Psi}_{l}(x, k)=e^{k_{l} x}+ \\
\left.\frac{1}{4 k^{3}} \int_{x}^{+\infty} \sum_{j: \operatorname{Re}\left(k_{j}\right)>\operatorname{Re}\left(k_{l}\right)} e^{k_{j}(x-y)}\left(k_{j}\right)^{2} u(y)+k_{j} u^{\prime}(y)+v(y)\right) \tilde{\Psi}_{l}(y, k) d y \\
-\frac{1}{4 k^{3}} \int_{-\infty}^{x} \sum_{j: \operatorname{Re}\left(k_{j}\right) \leq \operatorname{Re}\left(k_{l}\right)} e^{k_{j}(x-y)}\left(\left(k_{j}\right)^{2} u(y)+k_{j} u^{\prime}(y)+v(y)\right) \tilde{\Psi}_{l}(y, k) d y .
\end{gathered}
$$

If $k \in \gamma_{1}$ and $l=1$ or 4 then in the latter equation the sum in the second integral disappears and $\tilde{\Psi}_{1}(x, k)$ and $\tilde{\Psi}_{4}(x, k)$ satisfy Volterra's integral equation. This implies that these functions are analytic at $k=k_{*}$.

By using asymptotic formulae (2.2) and (2.8) we find that there is a natural relation between $\tilde{\Psi}_{1}(x, k)$ and $\tilde{\Psi}_{4}(x, k)$ and our basic functions $\Psi_{1}$ and $\Psi_{4}$

$$
\Psi_{1}(x, k)=a_{1}(k) \tilde{\Psi}_{1}(x, k), \quad \Psi_{4}(x, k)=a_{4}(k) \tilde{\Psi}_{4}(x, k) .
$$

Hence functions $\Psi_{1}(x, k)$ and $\Psi_{4}(x, k)$ have simple poles at point $k_{*}$. Using invariance under the transformation $k \mapsto i k$ we obtain the complete description of poles of functions $\Psi_{l}(x, k)$ :

$$
\begin{gathered}
\Psi_{1}(x, k)=e^{k x}\left(1+\frac{\alpha(x)}{-k-i k_{*}}+\frac{\beta(x)}{-k+k_{*}}\right), \\
\Psi_{2}(x, k)=e^{i k x}\left(1+\frac{\alpha(x)}{-i k-i k_{*}}+\frac{\beta(x)}{-i k+k_{*}}\right), \\
\Psi_{3}(x, k)=e^{-k x}\left(1+\frac{\alpha(x)}{k-i k_{*}}+\frac{\beta(x)}{k+k_{*}}\right),
\end{gathered}
$$




$$
\Psi_{4}(x, k)=e^{-i k x}\left(1+\frac{\alpha(x)}{i k-i k_{*}}+\frac{\beta(x)}{i k+k_{*}}\right) .
$$

We shall now compute the unknown functions $\alpha(x)$ and $\beta(x)$. We know that

$$
\Psi_{4}(x, k)=a_{4}(k) \tilde{\Psi}_{4}(x, k) .
$$

Clearly $\tilde{\Psi}_{4}\left(x, k_{*}\right)$ is a decaying function as $x \rightarrow-\infty$. Therefore, $\tilde{\Psi}_{4}$ can be expressed as a linear combination of functions $\Psi_{2}$ and $\Psi_{3}$

$$
\tilde{\Psi}_{4}\left(x, k_{*}\right)=z_{1} \Psi_{2}\left(x, k_{*}\right)+z_{2} \Psi_{3}\left(x, k_{*}\right) .
$$

So, we obtain that

$$
\operatorname{res}_{k=k_{*}} \Psi_{4}(x, k)=c \Psi_{2}\left(x, k_{*}\right)+d \Psi_{3}\left(x, k_{*}\right),
$$

where the constants $c=z_{1} \operatorname{res}_{k=k_{*}} a_{4}(k)$ and $d=z_{2} \operatorname{res}_{k=k_{*}} a_{4}(k)$. Similarly we find that there exist constants $\tilde{c}$ and $\tilde{d}$ such that

$$
\operatorname{res}_{k=k_{*}} \Psi_{1}(x, k)=\tilde{c} \Psi_{2}\left(x, k_{*}\right)+\tilde{d} \Psi_{3}\left(x, k_{*}\right) .
$$

Since $\tilde{\Psi}_{1}$ and $\tilde{\Psi}_{4}$ are linear independent we obtain that $c \tilde{d} \neq \tilde{c} d$. Selfadjointness of the operator $L$ and Lemma 2.2 imply that if $k \in \gamma_{1}$, then

$$
\Psi_{2}(x, k)=\overline{\Psi_{3}(x, i \bar{k})}, \quad \Psi_{1}(x, k)=\overline{\Psi_{4}(x, i \bar{k})} .
$$

Thus by using (3.7), (3.8) and (3.9) we find

$$
\tilde{c}=i \bar{d}, \quad \tilde{d}=i \bar{c} .
$$

3.3. Some relations between the coefficients $\mathbf{c}$ and $\mathbf{d}$. By using Lemma 2.1 we find that the Wronskian $\overline{\mathcal{W}_{3}\left[\Psi_{2}(x,-\bar{k}), \Psi_{3}(x,-\bar{k}), \Psi_{4}(x,-\bar{k})\right]}$ is a solution of the spectral equation (2.1). Comparing its behaviour as $x \rightarrow$ $+\infty$ with the asymptotic formulae (2.2) we obtain

$$
4 i k^{3} \Psi_{1}(x, k)=\overline{\mathcal{W}_{3}\left[\Psi_{2}(x,-\bar{k}), \Psi_{3}(x,-\bar{k}), \Psi_{4}(x,-\bar{k})\right]} .
$$

In particular, this implies

$$
4 i k_{*}^{3} \operatorname{res}_{k=k_{*}} \Psi_{1}(x, k)=\operatorname{res}_{k=k_{*}} \overline{\mathcal{W}_{3}\left[\Psi_{2}(x,-\bar{k}), \Psi_{3}(x,-\bar{k}), \Psi_{4}(x,-\bar{k})\right]} .
$$

Now, using identities (2.2), (3.7), (3.8) we obtain from (3.11) as $x \rightarrow+\infty$

$$
\begin{aligned}
& 4 i k_{*}^{3}\left(\tilde{c} e^{i k_{*} x}+\tilde{d} e^{-k_{*} x}\right)=\mathcal{W}_{3}\left[e^{i k_{*} x}, \tilde{c} e^{i k_{*} x}+\tilde{d} e^{-k_{*} x}, e^{-i k_{*} x}\right]+ \\
& +\mathcal{W}_{3}\left[e^{i k_{*} x}, e^{k_{*} x}, c e^{i k_{*} x}+d e^{-k_{*} x}\right]=\tilde{d} 4 i k_{*}^{3} e^{-k_{*} x}+d 4 k_{*}^{3} e^{i k_{*} x} .
\end{aligned}
$$

Finally, applying (3.10) we conclude that

$$
\operatorname{Re} d=0 \text {. }
$$


Inserting expressions (3.3)-(3.6) into (3.7) and (3.8) we finally obtain the system of two algebraic equations for coefficients $\alpha(x)$ and $\beta(x)$ :

$$
A(x)\left(\begin{array}{c}
\alpha(x) \\
\beta(x)
\end{array}\right)=\left(\begin{array}{c}
-c e^{i k_{*} x}-d e^{-k_{*} x} \\
-i \bar{d} e^{i k_{*} x}-i \bar{c} e^{-k_{*} x}
\end{array}\right)
$$

where

$$
A(x)=\left(\begin{array}{cc}
i e^{-i k_{*} x}-\frac{c e^{i k_{*} x}}{2 i k_{*}}+\frac{d e^{-k_{*} x}}{(1-i) k_{*}} & \frac{c e^{i k_{*} x}}{(1-i) k_{*}}+\frac{d e^{-k_{*} x}}{2 k_{*}} \\
-\frac{\overline{\bar{d}} e^{i k_{*} x}}{2 k_{*}}+\frac{i \bar{c} e^{-k_{*} x}}{(1-i) k_{*}} & e^{k_{*} x}+\frac{i \bar{d} e^{i k_{*} x}}{(1-i) k_{*}}+\frac{i \bar{c} e^{-k_{*} x}}{2 k_{*}}
\end{array}\right) .
$$

Let $d=i b, b \in \mathbb{R}$ and $k_{*}=(1+i) s_{*}$, then direct calculations show that $\operatorname{det} A(x)=i\left(e^{2 s_{*} x}+\frac{b^{2}-|c|^{2}}{8 s_{*}^{2}} e^{-2 s_{*} x}+\frac{b}{s_{*}}+\frac{|c|}{\sqrt{2} s_{*}} \sin \left(2 s_{*} x+\arg c+\frac{\pi}{4}\right)\right)$.

In order to find the family of reflectionless potentials corresponding to the eigenvalue $\lambda_{*}$ we have to find solutions $\alpha$ and $\beta$ of the equation (3.13). It is easy to verify that $\operatorname{det} A(x) \neq 0$ for all $x \in \mathbb{R}$ if and only if

$$
b>|c| \text {. }
$$

Example. If $c=0$ and $d=i 2 \sqrt{2} s_{*}$ then

$$
\begin{aligned}
& \alpha(x)=\frac{-\sqrt{2} s_{*}-(1+i) s_{*} e^{-2 x s_{*}}}{\cosh \left(2 x s_{*}\right)+\sqrt{2}}, \\
& \beta(x)=\frac{-\sqrt{2} s_{*}-(1-i) s_{*} e^{-2 x s_{*}}}{\cosh \left(2 x s_{*}\right)+\sqrt{2}} .
\end{aligned}
$$

Substituting $\Psi_{1}(x, k)$ given by (3.3) into the equation (2.1) we obtain potentials $u(x)$ and $v(x)$

$$
\begin{gathered}
u(x)=16 s_{*}^{2}\left(\sqrt{2} W(x)-W^{2}(x)\right), \\
v(x)=16 s_{*}^{4}\left(\sqrt{2} W(x)-12 W^{2}(x)+16 \sqrt{2} W^{3}(x)-8 W^{4}(x)\right),
\end{gathered}
$$

where

$$
W(x)=\frac{1}{\cosh \left(2 s_{*} x\right)+\sqrt{2}} .
$$

The same solution was found in [9] with the help of a Hirota-type equation.

The next theorem summarizes the main results obtained in this Section.

Theorem 3.1. Let $L$ be a fourth order differential operator defined by (2.1). Assume that $L$ has one negative eigenvalue $\lambda_{*}$. For an arbitrary family of parameters $c$ and $d, c \in \mathbb{C}, d=i b, b>|c|$, there exists a unique pair of real potentials $u$ and $v$, such that the operator $L$ is reflection free and has $\lambda_{*}$ as its negative eigenvalue of multiplicity two. 
Remark. The potentials $u$ and $v$ can be found explicitly via solutions $\alpha$ and $\beta$ of the algebraic equation (3.13). In general, $\alpha(x)$ and $\beta(x)$ and consequently $u(x)$ and $v(x)$ are rational functions of $e^{2 s_{*} x}, e^{-2 s_{*} x}, \cos \left(2 s_{*} x\right)$ and $\sin \left(2 s_{*} x\right)$.

\section{REFLECTIONLESS POTENTIALS GENERATED BY SEVERAL NEGATIVE EIGENVALUES}

4.1. Solutions corresponding to several eigenvalues. We now consider a more general situation. Namely, let a reflectionless operator $L$ has $N$ negative eigenvalues $\lambda_{*}^{n}=\left(k_{*}^{n}\right)^{4}, \quad n=1,2, \ldots N$.

In the previous Section we studied the properties of solutions $\Psi_{l}$ at $k_{*}$. Notice that such arguments (arguments concerning the behaviour of $\Psi$ near $k_{*}$ ) were local and have not assumed that there were no other singular points. Therefore we find that eigenvalues $\lambda_{*}^{n}$ are all of multiplicity two and that the corresponding eigenfunctions are $\Psi_{2}\left(x, k_{*}^{n}\right)$ and $\Psi_{3}\left(x, k_{*}^{n}\right)$. Similarly to (3.3)-(3.6) we obtain that the four independent solutions $\left\{\Psi_{l}(x, k)\right\}_{l=1}^{4}$ are given in the following form:

$$
\begin{aligned}
& \Psi_{1}(x, k)=e^{k x}\left(1+\sum_{n=1}^{N}\left(\frac{\alpha^{n}(x)}{-k-i k_{*}^{n}}+\frac{\beta^{n}(x)}{-k+k_{*}^{n}}\right)\right), \\
& \Psi_{2}(x, k)=e^{i k x}\left(1+\sum_{l=1}^{N}\left(\frac{\alpha^{n}(x)}{-i k-i k_{*}^{n}}+\frac{\beta^{n}(x)}{-i k+k_{*}^{n}}\right)\right), \\
& \Psi_{3}(x, k)=e^{-k x}\left(1+\sum_{l=1}^{N}\left(\frac{\alpha^{n}(x)}{k-i k_{*}^{n}}+\frac{\beta^{n}(x)}{k+k_{*}^{n}}\right)\right), \\
& \Psi_{4}(x, k)=e^{-i k x}\left(1+\sum_{n=1}^{N}\left(\frac{\alpha^{n}(x)}{i k-i k_{*}^{n}}+\frac{\beta^{n}(x)}{i k+k_{*}^{n}}\right)\right) .
\end{aligned}
$$

As we had before, the functions $\Psi_{j}(x, k)$ satisfy the identities

$$
\begin{aligned}
& \operatorname{res}_{k=k_{*}^{n}} \Psi_{4}(x, k)=c_{n} \Psi_{2}\left(x, k_{*}^{n}\right)+d_{n} \Psi_{3}\left(x, k_{*}^{n}\right), \\
& \operatorname{res}_{k=k_{*}^{n}} \Psi_{1}(x, k)=\tilde{c}_{n} \Psi_{2}\left(x, k_{*}^{n}\right)+\tilde{d}_{n} \Psi_{3}\left(x, k_{*}^{n}\right) .
\end{aligned}
$$

Besides, as in (3.10) and (3.12) we find

$$
\tilde{c}_{n}=i \bar{d}_{n}, \quad \tilde{d}_{n}=i \bar{c}_{n}, \quad \operatorname{Re} d_{n}=0 .
$$


4.2. Further properties of $c_{n}$ and $d_{n}$. For one-soliton solution the inequality (3.14) followed from the explicit expression for the determinant of the matrix $A(x)$. For a $\mathrm{N}$-soliton solution the corresponding determinant is too complicated. Therefore in order to prove that the corresponding system of equations is uniquely solvable we need two auxiliary statements.

Lemma 4.1. The constants $d_{n}$ and $c_{n}$ introduced in (4.5) satisfy the following relations

$$
d_{n}=i b_{n}, \quad b_{n}>0, \quad b_{n}>\left|c_{n}\right|, \quad n=1,2 \ldots N .
$$

Proof. Let us consider two functions $\Psi_{2}(x, k)$ and $\Psi_{3}(x, k)$ satisfying the spectral equation (2.1).

Differentiating the identity $L \Psi_{2}(x, k)=k^{4} \Psi_{2}(x, k)$ with respect to $k$ we arrive at

$$
L \dot{\Psi}_{2}(x, k)=4 k^{3} \Psi_{2}(x, k)+k^{4} \dot{\Psi}_{2}(x, k) .
$$

Therefore for a fixed $k=k_{*}^{n}$ we obtain

$$
\begin{gathered}
-\left.\mathcal{W}_{2}\left[\Psi_{3}\left(x, k_{*}^{n}\right), \dot{\Psi}_{2}\left(x, k_{*}^{n}\right)\right]\right|_{-\infty} ^{+\infty}=4\left(k_{*}^{n}\right)^{3} \int_{-\infty}^{+\infty} \Psi_{2}\left(x, k_{*}^{n}\right) \Psi_{3}\left(x, k_{*}^{n}\right) d x \\
=4\left(k_{*}^{n}\right)^{3} \int_{-\infty}^{+\infty}\left|\Psi_{2}\left(x, k_{*}^{n}\right)\right|^{2} d x
\end{gathered}
$$

where the latter equality follows from $\Psi_{2}(x, k)=\overline{\Psi_{3}(x, i \bar{k})}$.

From (4.2) and (4.3) we immediately find that

$$
\lim _{x \rightarrow+\infty} \mathcal{W}_{2}\left[\Psi_{3}\left(x, k_{*}^{n}\right), \dot{\Psi}_{2}\left(x, k_{*}^{n}\right)\right]=0 .
$$

Let us consider the behaviour of the Wronskian $\mathcal{W}_{2}\left[\Psi_{3}, \dot{\Psi}_{2}\right]$ at $-\infty$. According to (2.4), $\Psi_{2}(x, k)$ and its derivative with respect to $k$ have the following asymptotics as $x \rightarrow-\infty$ :

$\Psi_{2}(x, k)=\exp (i k x)\left(a_{2}(k)+o(1)\right), \quad \dot{\Psi}_{2}\left(x, k_{*}^{n}\right)=\exp \left(i k_{*}^{n} x\right)\left(\dot{a}_{2}\left(k_{*}^{n}\right)+o(1)\right)$.

Since $\Psi_{2}\left(x, k_{*}^{n}\right)$ and $\Psi_{3}\left(x, k_{*}^{n}\right)$ are two linear independent eigenfunctions they can be written as linear combinations of $\tilde{\Psi}_{1}$ and $\tilde{\Psi}_{4}$

$$
\begin{aligned}
& \Psi_{2}\left(x, k_{*}^{n}\right)=e_{1}^{n} \tilde{\Psi}_{1}\left(x, k_{*}^{n}\right)+e_{2}^{n} \tilde{\Psi}_{4}\left(x, k_{*}^{n}\right), \\
& \Psi_{3}\left(x, k_{*}^{n}\right)=\bar{e}_{1}^{n} \tilde{\Psi}_{4}\left(x, k_{*}^{n}\right)+\bar{e}_{2}^{n} \tilde{\Psi}_{1}\left(x, k_{*}^{n}\right),
\end{aligned}
$$

where

$$
\left|e_{1}^{n}\right| \neq\left|e_{2}^{n}\right|
$$

Finally we obtain

$$
-\left.\mathcal{W}_{2}\left[\Psi_{3}\left(x, k_{*}^{n}\right), \dot{\Psi}_{2}\left(x, k_{*}^{n}\right)\right]\right|_{-\infty} ^{+\infty}=i \bar{e}_{1}^{n} 4\left(k_{*}^{n}\right)^{3} \dot{a}_{2}\left(k_{*}^{n}\right)
$$


or

$$
i \bar{e}_{1}^{n} \dot{a}_{2}\left(k_{*}^{n}\right)=\int_{-\infty}^{+\infty}\left|\Psi_{2}\left(x, k_{*}^{n}\right)\right|^{2} d x
$$

Repeating this argument for $\Psi_{2}(x, k)$ instead of $\Psi_{3}(x, k)$, namely considering the Wronskian $\Psi_{2}(x, k)$ and $\dot{\Psi}_{2}(x, k)$, we obtain

$$
i e_{2}^{n} \dot{a}_{2}\left(k_{*}^{n}\right)=\int_{-\infty}^{+\infty}\left(\Psi_{2}\left(x, k_{*}^{n}\right)\right)^{2} d x
$$

Therefore (4.9), (4.10) together with (4.8) imply

$$
\left|e_{1}^{n}\right|>\left|e_{2}^{n}\right|
$$

By Lemma 2.4

$$
\begin{gathered}
\operatorname{res}_{k=k_{*}^{n}} \Psi_{4}(x, k)=\tilde{\Psi}_{4}\left(x, k_{*}^{n}\right) \operatorname{res}_{k=k_{*}^{n}} a_{4}(k) \\
=\tilde{\Psi}_{4}\left(x, k_{*}^{n}\right) \operatorname{res}_{k=k_{*}^{n}} \frac{1}{a_{2}(k)} .
\end{gathered}
$$

Moreover, from (4.6) and (4.7) we have

$$
\tilde{\Psi}_{4}\left(x, k_{*}^{n}\right)=\frac{1}{\left|e_{1}^{n}\right|^{2}-\left|e_{2}^{n}\right|^{2}}\left(-\bar{e}_{2}^{n} \Psi_{2}+e_{1}^{n} \Psi_{3}\right) .
$$

Equations (4.11) and (4.9) lead us to

$$
\operatorname{res}_{k=k_{*}^{n}} \Psi_{4}(x, k)=\frac{i \bar{e}_{1}^{n}}{\left(\left|e_{1}^{n}\right|^{2}-\left|e_{2}^{n}\right|^{2}\right)} \frac{\left(-\bar{e}_{2}^{n} \Psi_{2}+e_{1}^{n} \Psi_{3}\right)}{\int_{-\infty}^{+\infty}\left|\Psi_{2}\left(x, k_{*}^{n}\right)\right|^{2} d x} .
$$

Thus

$$
\begin{aligned}
& d_{n}=\frac{i\left|e_{1}^{n}\right|^{2}}{\left(\left|e_{1}^{n}\right|^{2}-\left|e_{2}^{n}\right|^{2}\right) \int_{-\infty}^{+\infty}\left|\Psi_{2}\left(x, k_{*}^{n}\right)\right|^{2} d x}, \\
& c_{n}=\frac{-i \bar{e}_{1}^{n} \bar{e}_{2}^{n}}{\left(\left|e_{1}^{n}\right|^{2}-\left|e_{2}^{n}\right|^{2}\right) \int_{-\infty}^{+\infty}\left|\Psi_{2}\left(x, k_{*}^{n}\right)\right|^{2} d x}
\end{aligned}
$$

and therefore $d_{n}=i b_{n}$ with some $b_{n}>0$ and $b_{n}>\left|c_{n}\right|, n=1,2, \ldots, N$. The proof is complete.

Inserting expressions (4.1)-(4.4) into (4.6) and (4.7) we obtain the system of $2 N$ algebraic equations for the coefficients $\alpha^{n}(x)$ and $\beta^{n}(x)$ :

$$
\begin{gathered}
-i e^{-i k_{*}^{j} x} \alpha^{j}(x)=c_{j} e^{i k_{*}^{j} x}\left(1+\sum_{n=1}^{N}\left(\frac{\alpha^{n}(x)}{-i k_{*}^{j}-i k_{*}^{n}}+\frac{\beta^{n}(x)}{-i k_{*}^{j}+k_{*}^{n}}\right)\right) \\
+d_{j} e^{-k_{*}^{j} x}\left(1+\sum_{n=1}^{N}\left(\frac{\alpha^{n}(x)}{k_{*}^{j}-i k_{*}^{n}}+\frac{\beta^{n}(x)}{k_{*}^{j}+k_{*}^{n}}\right)\right),
\end{gathered}
$$




$$
\begin{gathered}
-e^{k_{*}^{j} x} \beta^{j}(x)=i \overline{d_{j}} e^{i k_{*}^{j} x}\left(1+\sum_{n=1}^{N}\left(\frac{\alpha^{n}(x)}{-i k_{*}^{j}-i k_{*}^{n}}+\frac{\beta^{n}(x)}{-i k_{*}^{j}+k_{*}^{n}}\right)\right) \\
+i \overline{c_{j}} e^{-k_{*}^{j} x}\left(1+\sum_{n=1}^{N}\left(\frac{\alpha^{n}(x)}{k_{*}^{j}-i k_{*}^{n}}+\frac{\beta^{n}(x)}{k_{*}^{j}+k_{*}^{n}}\right)\right) .
\end{gathered}
$$

4.3. Solvability of algebraic equations. Solvability of this system depends on it's determinant $\Delta(x)$.

Lemma 4.2. Determinant $\Delta(x)$ of the matrix described by the above equations is non-degenerate for all $x \in \mathbb{R}$.

Proof. Let us assume that $\Delta\left(x_{0}\right)=0$ for some $x_{0} \in \mathbb{R}$. Then for this $x_{0}$ we have a non-trivial solution $\left(\alpha_{0}^{n}, \beta_{0}^{n}\right)$ of our algebraic homogeneous system of equations

$$
\begin{gathered}
-i e^{-i k_{*}^{j} x_{0}} \alpha_{0}^{j}=c_{j} e^{i k_{*}^{j} x_{0}} \sum_{n=1}^{N}\left(\frac{\alpha_{0}^{n}}{-i k_{*}^{j}-i k_{*}^{n}}+\frac{\beta_{0}^{n}}{-i k_{*}^{j}+k_{*}^{n}}\right) \\
+d_{j} e^{-k_{*}^{j} x_{0}} \sum_{n=1}^{N}\left(\frac{\alpha_{0}^{n}}{k_{*}^{j}-i k_{*}^{n}}+\frac{\beta_{0}^{n}}{k_{*}^{j}+k_{*}^{n}}\right), \\
-e^{k_{*}^{j} x_{0}} \beta_{0}^{j}=i \overline{d_{j}} e^{i k_{*}^{j} x_{0}} \sum_{n=1}^{N}\left(\frac{\alpha_{0}^{n}}{-i k_{*}^{j}-i k_{*}^{n}}+\frac{\beta_{0}^{n}}{-i k_{*}^{j}+k_{*}^{n}}\right)+ \\
+i \overline{c_{j}} e^{-k_{*}^{j} x_{0}} \sum_{n=1}^{N}\left(\frac{\alpha_{0}^{n}}{k_{*}^{j}-i k_{*}^{n}}+\frac{\beta_{0}^{n}}{k_{*}^{j}+k_{*}^{n}}\right)
\end{gathered}
$$

Let us consider four functions

$$
\begin{gathered}
\Psi_{1}^{0}(k)=e^{k x_{0}} \sum_{n=1}^{N}\left(\frac{\alpha_{0}^{n}}{-k-i k_{*}^{n}}+\frac{\beta_{0}^{n}}{-k+k_{*}^{n}}\right), \\
\Psi_{2}^{0}(k)=e^{i k x_{0}} \sum_{n=1}^{N}\left(\frac{\alpha_{0}^{n}}{-i k-i k_{*}^{n}}+\frac{\beta_{0}^{n}}{-i k+k_{*}^{n}}\right), \\
\Psi_{3}^{0}(k)=e^{-k x_{0}} \sum_{n=1}^{N}\left(\frac{\alpha_{0}^{n}}{k-i k_{*}^{n}}+\frac{\beta_{0}^{n}}{k+k_{*}^{n}}\right), \\
\Psi_{4}^{0}(k)=e^{-i k x_{0}} \sum_{n=1}^{N}\left(\frac{\alpha_{0}^{n}}{i k-i k_{*}^{n}}+\frac{\beta_{0}^{n}}{i k+k_{*}^{n}}\right) .
\end{gathered}
$$


It follows from Lemma 2.2 that $\Psi_{1}(x, i s)=\overline{\Psi_{3}(x, i s)}, s \in \mathbb{R}$. Thus

$$
I:=\int_{-i \infty}^{+i \infty} \Psi_{1}^{0}(k) \Psi_{3}^{0}(k) d k=\int_{-i \infty}^{+i \infty}\left|\Psi_{1}^{0}(k)\right|^{2} d k .
$$

We now observe that the value of the integral $I=i p, p \geq 0$, and it can be zero only when both constants $\alpha_{0}^{n}, \beta_{0}^{n}$ are zero.

On the other hand we can calculate $I$ with the help of residues

$$
I=-2 \pi i \sum_{n=1}^{N}\left(\Psi_{3}^{0}\left(k_{*}^{n}\right) \operatorname{res}_{k=k_{*}^{n}} \Psi_{1}^{0}+\Psi_{3}^{0}\left(-i k_{*}^{n}\right) \operatorname{res}_{k=-i k_{*}^{n}} \Psi_{1}^{0}\right) .
$$

By analogy with (3.7) and (3.8) we can obtain

$$
\begin{gathered}
\operatorname{res}_{k=k_{*}^{n}} \Psi_{4}^{0}(k)=c_{n} \Psi_{2}^{0}\left(k_{*}^{n}\right)+d_{n} \Psi_{3}^{0}\left(k_{*}^{n}\right) \\
\operatorname{res}_{k=k_{*}^{n}} \Psi_{1}^{0}(k)=\tilde{c}_{n} \Psi_{2}^{0}\left(k_{*}^{n}\right)+\tilde{d}_{n} \Psi_{3}^{0}\left(k_{*}^{n}\right) .
\end{gathered}
$$

Hence

$$
I=-2 \pi i \sum_{n=1}^{N}\left(2 b_{n}\left|\Psi_{2}^{0}\left(k_{*}^{n}\right)\right|^{2}+2 \operatorname{Im}\left(c_{n}\left(\Psi_{2}^{0}\left(k_{*}^{n}\right)\right)^{2}\right)\right) .
$$

Applying Lemma 4.1 we obtain

$$
I=-i \hat{p}, \quad \hat{p}>0,
$$

which contradicts the equality $I=i p, p \geq 0$.

We have now proved the following result.

Theorem 4.1. Let $L$ be a fourth order differential operator defined by (2.1). Assume that $L$ has $N$ negative eigenvalues $\lambda_{*}^{n}, n=1,2, \ldots, N$. Then for an arbitrary family of parameters $c_{n}$ and $d_{n}, c_{n} \in \mathbb{C}, d_{n}=i b_{n}, b_{n}>$ $\left|c_{n}\right|, n=1,2, \ldots, N$, there exists a unique pair of real potentials $u$ and $v$, such that the operator $L$ is reflection free. Each of the eigenvalues $\lambda_{*}^{n}$ is of multiplicity two.

\section{REFLECTIONLESS POTENTIALS CORRESPONDING TO A POSITIVE EIGENVALUE}

The scheme used in the previous section can be applied to constructing reflectionless potentials corresponding to a positive eigenvalue belonging to the continuous spectrum. Note that for a fourth order operator $L$ the eigenvalues embedded into the positive continuous spectrum are always of multiplicity one.

Let $k_{*}^{4}, k_{*}>0$ be such an eigenvalue. Then function $a(k)=a_{2}(k) a_{3}(k)$ has a simple zero at the point $k_{*}$. Using Lemma 2.3 and $a_{2}(k)=\overline{a_{4}(k)}$, for $k>0$, (see Lemma 2.4) we obtain that $a_{2}(k)$ and $a_{4}(k)$ are both analytic 
and nonzero at $k_{*}$, while $a_{3}(k)$ has simple zero at $k_{*}$ and $a_{1}(k)$ has simple pole at $k_{*}$. Using asymptotics with respect to $k \rightarrow \infty$ we arrive at the following expression for $\Psi_{1}(x, k)$ :

$$
\Psi_{1}(x, k)=e^{k x}\left(1+\frac{\gamma(x)}{k-k_{*}}\right),
$$

and using invariance under $k \mapsto i k$ we get

$$
\begin{gathered}
\Psi_{2}(x, k)=e^{i k x}\left(1+\frac{\gamma(x)}{i k-k_{*}}\right), \\
\Psi_{3}(x, k)=e^{-k x}\left(1+\frac{\gamma(x)}{-k-k_{*}}\right), \\
\Psi_{4}(x, k)=e^{-i k x}\left(1+\frac{\gamma(x)}{-i k-k_{*}}\right) .
\end{gathered}
$$

Let $\tilde{\Psi}_{1}(x, k)$ be the solution of (2.1) such that

$$
\begin{gathered}
\tilde{\Psi}_{1}(x, k)=e^{k x}(1+o(1)), x \rightarrow-\infty \\
\tilde{\Psi}_{1}(x, k)=e^{k x} O(1), x \rightarrow+\infty .
\end{gathered}
$$

Then by using uniqueness (cf. (3.2))

$$
\Psi_{1}(x, k)=a_{1}(k) \tilde{\Psi}_{1}(x, k) .
$$

Function $\tilde{\Psi}_{1}(x, k)$ satisfies a Volterra integral equation for $\{|\operatorname{Im} k|<\operatorname{Re} k\}$ and therefore is analytic at $k_{*}$. Moreover, functions $\tilde{\Psi}_{1}\left(x, k_{*}\right)$ and $\Psi_{3}\left(x, k_{*}\right)$ are the only decaying solutions at $-\infty$ and $+\infty$ respectively. Since $k_{*}^{4}$ is an eigenvalue of multiplicity one we obtain that there exists a constant $\hat{c} \neq 0$ such that

$$
\tilde{\Psi}_{1}\left(x, k_{*}\right)=\hat{c} \Psi_{3}\left(x, k_{*}\right),
$$

and therefore

$$
\operatorname{res}_{k=k_{*}} \Psi_{1}(x, k)=c \Psi_{3}\left(x, k_{*}\right),
$$

where $c=\hat{c}$ res $_{k=k_{*}} a_{1}(k)$. Substituting expressions for $\Psi_{1}(x, k), \Psi_{3}(x, k)$ into (5.1) we obtain

$$
\gamma(x)=c\left(e^{2 k_{*} x}+\frac{c}{2 k_{*}}\right)^{-1} .
$$

Obviously, if $c>0$ then $\gamma(x) \neq 0$ for all $x \in \mathbb{R}$.

If, for example, $c=2 k_{*}$ then by inserting $\Psi_{3}(x, k)$ into equation (2.1) we obtain the following pair of reflectionless potentials

$$
u_{k_{*}}(x)=\frac{4 k_{*}^{2}}{\cosh ^{2}\left(k_{*} x\right)}, \quad v_{k_{*}}(x)=\frac{8 k_{*}^{4} \sinh ^{2}\left(k_{*} x\right)}{\cosh ^{4}\left(k_{*} x\right)} .
$$


Such a pair corresponds to a single positive eigenvalue $k_{*}^{4}$ with an eigenfunction $\cosh ^{-1}\left(k_{*} x\right)$. Note that the operator $L$ with potentials $u_{k_{*}}(x), v_{k_{*}}(x)$ can be obtained as a square of the Schrödinger operator $H=-d^{2} / d x^{2}-2 k_{*}^{2} \cosh ^{-2}\left(k_{*} x\right)$.

The same result also holds true in a more general case.

Theorem 5.1. Let $L$ be an operator (1.1) with real-valued reflectionless potentials $u$ and $v$ satisfying (1.2). Let the operator $L$ has only spectral singularities corresponding to the positive eigenvalues $\left(k_{*}^{n}\right)^{4}, k_{*}^{n}>0, n=$ $1, \ldots, N$. Then this operator is equal to the square of a Schrödinger operator with some real-valued reflectionless potential $w$ corresponding to negative eigenvalues $-\left(k_{*}^{n}\right)^{2}, n=1, \ldots, N$.

Proof. Repeating arguments used for the case of one positive eigenvalue we obtain the following expressions for $\Psi_{1}(x, k), \Psi_{3}(x, k)$

$$
\begin{gathered}
\Psi_{1}(x, k)=e^{k x}\left(1+\sum_{n=1}^{N} \frac{\gamma_{n}(x)}{k-k_{*}^{n}}\right), \\
\Psi_{3}(x, k)=e^{-k x}\left(1+\sum_{n=1}^{N} \frac{\gamma_{n}(x)}{-k-k_{*}^{n}}\right),
\end{gathered}
$$

satisfying the system of equations

$$
\operatorname{res}_{k=k_{*}^{n}} \Psi_{1}(x, k)=c_{n} \Psi_{3}\left(x, k_{*}^{n}\right), \quad c_{n} \neq 0, n=1, \ldots N .
$$

Note now that the equations (5.3) - (5.5) coincide with the corresponding expressions when solving the inverse problem for a Schrödinger equation, see [5] ([7]). Therefore there is a reflectionless real-valued potential $w$ for which the Schrödinger operator

$$
H \Psi(x, k):=\left(-\frac{d^{2}}{d x^{2}}+w(x)\right) \Psi(x, k)=-k^{2} \Psi(x, k)
$$

has negative eigenvalues $-\left(k_{*}^{n}\right)^{2}, n=1, \ldots, N$. The condition of nonsingularity of the potential $w$ implies $c_{n}>0$ for all $n=1, \ldots, N$. We also have

$$
H^{2} \Psi_{3}(x, k)=k^{4} \Psi_{3}(x, k)
$$

and thus $H^{2}=L$.

\section{NON-SPECTRAL SINGULARITIES}

Let us now consider reflectionless operator $L$ for which the corresponding Riemann-Hilbert problem has non-spectral singularities. For such a problem singular points of the functions $a_{l}(k)$ satisfy some symmetries. 
6.1. Structure of poles and zeros of solutions $\Psi_{1}$. Let, for example, $a_{1}(k)$ has a simple pole at point $k_{*} \in \Omega_{1}$. Then (see (2.9)) the function $a_{3}(k)$ has a simple zero at $k_{*}$.

Now, we would like to show that $a_{2}(k)$ has a simple pole and consequently (see (2.9)) $a_{4}(k)$ has a simple zero at $k_{*}$. In order to prove this we need the following below two simple statements.

Lemma 6.1. There exist solutions $\left\{\varphi_{l}(x, k)\right\}, l=1,2,3,4$, of equation (2.1) which are analytic in $k \in \Omega_{1}$ and satisfy the boundary conditions

$$
\varphi_{l}(x, k)=e^{k_{l} x}(1+o(1)), \quad x \rightarrow+\infty, \quad l=1,2,3,4 .
$$

For a proof of this Lemma see, for example, [6].

The following fact is trivial.

Lemma 6.2. Let $k \in \Omega_{1}$. Then for any solution $\Phi(x, k)$ of the equation (2.1) there exists a pair $\left(l, l^{\prime}\right) l, l^{\prime} \in\{1,2,3,4\}$, such that $\Phi(x, k)$ satisfies the boundary conditions

$$
\begin{aligned}
& \Phi(x, k)=e^{k_{l} x}\left(\varrho_{1}(k)+o(1)\right), \quad x \rightarrow+\infty, \\
& \Phi(x, k)=e^{k_{l^{\prime}} x}\left(\varrho_{2}(k)+o(1)\right), \quad x \rightarrow-\infty,
\end{aligned}
$$

with some $\varrho_{1} \neq 0, \varrho_{2} \neq 0$.

Comparing the asymptotics at $+\infty$ we find that $\varphi_{3}(x, k)=\Psi_{3}(x, k)$ for $k \in \Omega_{1}$. From Lemmas 6.1 and 6.2 we have

$$
\varphi_{2}(x, k)=e^{-k x}(\varrho(k)+o(1)), \quad x \rightarrow-\infty, \quad k \in \Omega_{1},
$$

where $\varrho(k)$ is an analytic in $k$ function. Here we do not know a priori that $\varrho \neq 0$. From (2.2), (2.8), (6.1) and (6.2) we obtain that

$$
\Psi_{2}(x, k)=\varphi_{2}(x, k)-\frac{\varrho(k)}{a_{3}(k)} \Psi_{3}(x, k), \quad k \in \Omega_{1} .
$$

Let us show that

$$
\varrho\left(k_{*}\right) \neq 0 .
$$

Indeed, if we assume that $\varrho\left(k_{*}\right)=0$, then $\Psi_{2}(x, k)$ is regular at $k_{*}$. Clearly, both $\Psi_{2}\left(x, k_{*}\right)$ and $\Psi_{3}\left(x, k_{*}\right)$ are exponentially decaying functions as $x \rightarrow+\infty$. From (2.8) it follows that $\Psi_{2}\left(x, k_{*}\right)=O\left(e^{i k_{*} x}\right)$. Since $a_{3}\left(k_{*}\right)=0$ then by using (2.8) and Lemma 6.2 we also obtain that $\Psi_{3}\left(x, k_{*}\right)=O\left(e^{i k_{*} x}\right)$. This means that there is a linear combination of $\Psi_{2}\left(x, k_{*}\right)$ and $\Psi_{3}\left(x, k_{*}\right)$ which crosses out the terms $O\left(e^{i k_{*} x}\right)$ and therefore exponentially decays as $x \rightarrow-\infty$. Thus, $k_{*}^{4}$ must be an eigenvalue of $L$ and this contradicts the selfadjointness of $L$. 
From (2.8) and (6.3), (6.4) we obtain that $a_{2}(k)$ has a simple pole at $k_{*}$. Using invariance under $k \mapsto i k$ we arrive at a complete picture of poles for functions $\Psi_{l}(x, k)$ :

$$
\Psi_{1}(x, k)=e^{k x}\left(1+\frac{\alpha(x)}{k-k_{*}}+\frac{\beta(x)}{k-i k_{*}}+\frac{\gamma(x)}{k-\bar{k}_{*}}+\frac{\delta(x)}{k+i \bar{k}_{*}}\right),
$$

$$
\Psi_{2}(x, k)=e^{i k x}\left(1+\frac{\alpha(x)}{i k-k_{*}}+\frac{\beta(x)}{i k-i k_{*}}+\frac{\gamma(x)}{i k-\bar{k}_{*}}+\frac{\delta(x)}{i k+i \bar{k}_{*}}\right),
$$

$$
\Psi_{3}(x, k)=e^{-k x}\left(1+\frac{\alpha(x)}{-k-k_{*}}+\frac{\beta(x)}{-k-i k_{*}}+\frac{\gamma(x)}{-k-\bar{k}_{*}}+\frac{\delta(x)}{-k+i \bar{k}_{*}}\right),
$$

$\Psi_{4}(x, k)=e^{-i k x}\left(1+\frac{\alpha(x)}{-i k-k_{*}}+\frac{\beta(x)}{-i k-i k_{*}}+\frac{\gamma(x)}{-i k-\bar{k}_{*}}+\frac{\delta(x)}{-i k+i \bar{k}_{*}}\right)$.

From (6.3) we immediately observe that

$$
\operatorname{res}_{k=k_{*}} \Psi_{2}(x, k)=d_{1} \Psi_{3}\left(x, k_{*}\right) .
$$

Similar arguments give us three more identities

$$
\begin{aligned}
& \operatorname{res}_{k=k_{*}} \Psi_{1}(x, k)=d_{3} \Psi_{4}\left(x, k_{*}\right), \\
& \operatorname{res}_{k=\bar{k}_{*}} \Psi_{4}(x, k)=d_{2} \Psi_{3}\left(x, \bar{k}_{*}\right), \\
& \operatorname{res}_{k=\bar{k}_{*}} \Psi_{1}(x, k)=d_{4} \Psi_{2}\left(x, \bar{k}_{*}\right) .
\end{aligned}
$$

By Lemma 2.2 we have

$$
d_{2}=\bar{d}_{1}, \quad d_{4}=\bar{d}_{3}
$$

Lemma 2.1 and asymptotics at $+\infty$ (2.2) imply (cf. (3.11))

$$
4 i k_{*}^{3} \operatorname{res}_{k=k_{*}} \Psi_{1}(x, k)=\operatorname{res}_{k=k_{*}} \overline{W\left[\Psi_{2}(x,-\bar{k}), \Psi_{3}(x,-\bar{k}), \Psi_{4}(x,-\bar{k})\right]} .
$$

Combining the latter identity with (2.2), (6.9) and (6.10) and computing the residue of the Wronskian we obtain

$$
d_{3}=i d_{1} .
$$

If we insert the expressions (6.5)-(6.8) into (6.9)-(6.12) we arrive at a system of four algebraic equations for the coefficients $\alpha(x), \beta(x), \gamma(x)$ and $\delta(x)$

$$
A \zeta=\mathbf{i}_{0},
$$


where

$$
\begin{gathered}
A(x)= \\
=\left(\begin{array}{cccc}
\frac{1}{k_{*}(1+i)}+\frac{e^{k_{*} x(1+i)}}{d_{3}} & \frac{-i}{2 k_{*}} & \frac{1}{i k_{*}+\overline{k_{*}}} & \frac{-i}{k_{*}-\overline{k_{*}}} \\
\frac{1}{2 k_{*}} & \frac{1}{k_{*}(1+i)}-i \frac{e^{k * x(1+i)}}{d_{1}} & \frac{1}{k_{*}+\overline{k_{*}}} & \frac{1}{k_{*}-i \overline{k_{*}}} \\
\frac{1}{k_{*}-i \overline{k_{*}}} & \frac{-i}{k_{*}-\overline{k_{*}}} & \frac{1}{\overline{k_{*}}(1-i)}+\frac{e^{\bar{k}_{*}} x(1-i)}{d_{4}} & \frac{i}{2 \overline{k_{*}}} \\
\frac{1}{k_{*}+\overline{k_{*}}} & \frac{1}{i k_{*}+\overline{k_{*}}} & \frac{i}{2 \overline{k_{*}}} & \frac{1}{\overline{k_{*}}(1-i)}+i \frac{e^{\overline{k_{*}} x(1-i)}}{d_{2}}
\end{array}\right) \\
\zeta=\left(\begin{array}{c}
\alpha \\
\beta \\
\gamma \\
\delta
\end{array}\right), \quad \mathbf{i}_{0}=\left(\begin{array}{l}
1 \\
1 \\
1 \\
1
\end{array}\right) .
\end{gathered}
$$

6.2. Solvability. In order to prove that there exists a unique solution of this system of equations we consider the following statement.

Lemma 6.3. Determinant $\Delta(x)=\operatorname{det} A(x)$ is a non-degenerate function for all $x \in \mathbb{R}$.

Proof. The proof is based on a theorem of uniqueness for the corresponding Riemann-Hilbert problem (see [1], [10]). Some of the arguments below were already used in Lemmas 4.1 and 4.2.

Let us assume that there is $x_{0}$ such that

$$
\Delta\left(x_{0}\right)=0 .
$$

Then at this point we can find a nontrivial solution $\alpha_{0}, \beta_{0}, \gamma_{0}$, and $\delta_{0}$ of the homogeneous system of equations

$$
A \zeta=\mathbf{0}
$$

Let us introduce the following four functions:

$$
\begin{gathered}
\Psi_{1}^{0}(k)=e^{k x_{0}}\left(\frac{\alpha_{0}}{k-k_{*}}+\frac{\beta_{0}}{k-i k_{*}}+\frac{\gamma_{0}}{k-\bar{k}_{*}}+\frac{\delta_{0}}{k+i \bar{k}_{*}}\right), \\
\Psi_{2}^{0}(k)=e^{i k x_{0}}\left(\frac{\alpha_{0}}{i k-k_{*}}+\frac{\beta_{0}}{i k-i k_{*}}+\frac{\gamma_{0}}{i k-\bar{k}_{*}}+\frac{\delta_{0}}{i k+i \bar{k}_{*}}\right), \\
\Psi_{3}^{0}(k)=e^{-k x_{0}}\left(\frac{\alpha_{0}}{-k-k_{*}}+\frac{\beta_{0}}{-k-i k_{*}}+\frac{\gamma_{0}}{-k-\bar{k}_{*}}+\frac{\delta_{0}}{-k+i \bar{k}_{*}}\right), \\
\Psi_{4}^{0}(k)=e^{-i k x_{0}}\left(\frac{\alpha_{0}}{-i k-k_{*}}+\frac{\beta_{0}}{-i k-i k_{*}}+\frac{\gamma_{0}}{-i k-\bar{k}_{*}}+\frac{\delta_{0}}{-i k+i \bar{k}_{*}}\right) .
\end{gathered}
$$

By using the fact that $\left(\alpha_{0}, \beta_{0}, \gamma_{0}, \delta_{0}\right)$ is a solution of (6.15) we conclude that $\Psi_{l}$ satisfy the equations (6.9) - (6.12) and therefore

$$
\operatorname{res}_{k=k_{*}} \Psi_{2}^{0}(k)=d_{1} \Psi_{3}^{0}\left(k_{*}\right),
$$




$$
\begin{gathered}
\operatorname{res}_{k=k_{*}} \Psi_{1}^{0}(k)=d_{3} \Psi_{4}^{0}\left(k_{*}\right), \\
\operatorname{res}_{k=\bar{k}_{*}} \Psi_{4}^{0}(k)=d_{2} \Psi_{3}^{0}\left(\bar{k}_{*}\right), \\
\operatorname{res}_{k=\bar{k}_{*}} \Psi_{1}^{0}(k)=d_{4} \Psi_{2}^{0}\left(\bar{k}_{*}\right) .
\end{gathered}
$$

As in Lemma 4.2 we find that

$$
I:=\int_{-i \infty}^{+i \infty} \Psi_{1}^{0}(k) \Psi_{3}^{0}(k) d k=\int_{-i \infty}^{+i \infty}\left|\Psi_{1}^{0}(k)\right|^{2} d k .
$$

The latter integral can be zero only if all the constants $\alpha_{0}, \beta_{0}, \gamma_{0}$ and $\delta_{0}$ are zero. Computing $I$ with help of residues we find

$$
\begin{gathered}
I=-2 \pi i\left(\Psi_{3}^{0}\left(k_{*}\right) \operatorname{res}_{k=k_{*}} \Psi_{1}^{0}+\Psi_{3}^{0}\left(\bar{k}_{*}\right) \operatorname{res}_{k=\bar{k}_{*}} \Psi_{1}^{0}+\Psi_{1}^{0}\left(i \bar{k}_{*}\right) \operatorname{res}_{k=i \bar{k}_{*}} \Psi_{3}^{0}\right. \\
\left.+\Psi_{1}^{0}\left(-i k_{*}\right) \operatorname{res}_{k=-i k_{*}} \Psi_{3}^{0}\right) .
\end{gathered}
$$

Equations (6.16) - (6.19) imply

$$
\begin{gathered}
\operatorname{res}_{k=k_{*}} \Psi_{1}^{0}=d_{3} \Psi_{4}^{0}\left(k_{*}\right), \\
\operatorname{res}_{k=\bar{k}_{*}} \Psi_{1}^{0}=d_{4} \Psi_{2}^{0}\left(\bar{k}_{*}\right), \\
\operatorname{res}_{k=i \bar{k}_{*}} \Psi_{3}^{0}=i \operatorname{res}_{k=\bar{k}_{*}} \Psi_{4}^{0}=i d_{2} \Psi_{3}^{0}\left(\bar{k}_{*}\right), \\
\operatorname{res}_{k=-i k_{*}} \Psi_{3}^{0}=-i \operatorname{res}_{k=k_{*}} \Psi_{2}^{0}=-i d_{1} \Psi_{3}^{0}\left(k_{*}\right) .
\end{gathered}
$$

Therefore

$$
\begin{aligned}
I= & -2 \pi i\left(d_{3} \Psi_{3}^{0}\left(k_{*}\right) \Psi_{4}^{0}\left(k_{*}\right)+d_{4} \Psi_{3}^{0}\left(\bar{k}_{*}\right) \Psi_{2}^{0}\left(\bar{k}_{*}\right)\right. \\
& \left.+i d_{2} \Psi_{2}^{0}\left(\bar{k}_{*}\right) \Psi_{3}^{0}\left(\bar{k}_{*}\right)-i d_{1} \Psi_{4}^{0}\left(k_{*}\right) \Psi_{3}^{0}\left(k_{*}\right)\right) .
\end{aligned}
$$

Taking into account (6.13) and (6.14) we arrive at $I=0$ which gives us a contradiction. Lemma is proved.

Remark 1. Cancellations appearing in the right hand side of (6.20) has been already observed in [1] in a somewhat more general situations.

Remark 2. In this Section we consider only a one-soliton case. However, it is not difficult to generalize this approach to the case when there are $2 \mathrm{~N}$ non-spectral singularities (cf. Section 4).

Namely, let $\left\{k_{*}^{n}\right\}_{n=1}^{N}$ is a set of points from sector $\Omega_{1}$. Then the solutions $\Psi_{j}$ can be represented as follows:

$$
\begin{aligned}
& \Psi_{1}(x, k)=e^{k x}\left(1+\sum_{n=1}^{N}\left(\frac{\alpha^{n}(x)}{k-k_{*}^{n}}+\frac{\beta^{n}(x)}{k-i k_{*}^{n}}+\frac{\gamma^{n}(x)}{k-\bar{k}_{*}^{n}}+\frac{\delta^{n}(x)}{k+i \bar{k}_{*}^{n}}\right)\right), \\
& \Psi_{2}(x, k)=e^{i k x}\left(1+\sum_{n=1}^{N}\left(\frac{\alpha^{n}(x)}{i k-k_{*}^{n}}+\frac{\beta^{n}(x)}{i k-i k_{*}^{n}}+\frac{\gamma^{n}(x)}{i k-\bar{k}_{*}^{n}}+\frac{\delta^{n}(x)}{i k+i \bar{k}_{*}^{n}}\right)\right),
\end{aligned}
$$




$$
\begin{aligned}
& \Psi_{3}(x, k)=e^{-k x}\left(1+\sum_{n=1}^{N}\left(\frac{\alpha^{n}(x)}{-k-k_{*}^{n}}+\frac{\beta^{n}(x)}{-k-i k_{*}^{n}}+\frac{\gamma^{n}(x)}{-k-\bar{k}_{*}^{n}}+\frac{\delta^{n}(x)}{-k+i \bar{k}_{*}^{n}}\right)\right), \\
& \Psi_{4}(x, k)=e^{-i k x}\left(1+\sum_{n=1}^{N}\left(\frac{\alpha^{n}(x)}{-i k-k_{*}^{n}}+\frac{\beta^{n}(x)}{-i k-i k_{*}^{n}}+\frac{\gamma^{n}(x)}{-i k-\bar{k}_{*}^{n}}+\frac{\delta^{n}(x)}{-i k+i \bar{k}_{*}^{n}}\right)\right) .
\end{aligned}
$$

Therefore

with constants

$$
\begin{aligned}
& \operatorname{res}_{k=k_{*}^{n}} \Psi_{2}(x, k)=d_{1}^{n} \Psi_{3}\left(x, k_{*}^{n}\right), \\
& \operatorname{res}_{k=k_{*}^{n}} \Psi_{1}(x, k)=d_{3}^{n} \Psi_{4}\left(x, k_{*}^{n}\right), \\
& \operatorname{res}_{k=\bar{k}_{*}^{n}} \Psi_{4}(x, k)=d_{2}^{n} \Psi_{3}\left(x, \bar{k}_{*}^{n}\right), \\
& \operatorname{res}_{k=\bar{k}_{*}^{n}} \Psi_{1}(x, k)=d_{4}^{n} \Psi_{2}\left(x, \bar{k}_{*}^{n}\right) .
\end{aligned}
$$

$$
d_{2}^{n}=\bar{d}_{1}^{n}, \quad d_{4}^{n}=\bar{d}_{3}^{n}, \quad d_{3}^{n}=i d_{1}^{n} .
$$

Using these identities we can obtain a system of equations for the functions $\alpha^{n}(x), \beta^{n}(x), \gamma^{n}(x)$, and $\delta^{n}(x)(n=1,2,3 \ldots N)$. Solvability of this system can be proven in the same way as in Lemma 6.3.

Remark 3. It has become clear that the existence of non-spectral solitons is important for analyzing trace formulae for a fourth order differential operator. In particular, this explains the main result of the paper [3], where the authors construct a counter example to some functional inequality.

Let

$$
Q[u, v]=
$$

$7 \cdot 2^{-11} \int_{\mathbb{R}}\left(48 v^{2}+\frac{5}{4} u^{4}-12 u^{2} v-40 u^{\prime \prime} v+\frac{13}{2} u^{2} u^{\prime \prime}+9 u^{\prime \prime 2}\right) d x$.

The expression appearing in the right hand side of (6.21) is a point-wise quadratic form of $\left(u^{2}, u^{\prime \prime}, v\right)$. This quadratic form has two positive eigenvalues and one relatively small negative eigenvalue. There was a hope that after integration this quadratic form, the functional $Q$ would become nonnegative. However, it has been recently shown in [3] that there is a pair $(u, v)$ which violate such a positivity.

Let now $(u, v)$ be a pair of reflectionless potentials corresponding a nonspectral singularity $k_{*} \in \Omega_{1}$. Assume also for simplicity that $L$ does not have any other spectral or non-spectral singularities. In this case one can show (see [13]) that the following trace formula is true

$$
Q[u, v]=\operatorname{Re} k_{*}^{7}+\operatorname{Im} k_{*}^{7} .
$$

This immediately implies that if $3 \pi / 28<\arg k_{*}<\pi / 4$, then the right hand side of the latter equality is negative. In particular, this give an example of exponentially decaying potentials $u$ and $v$ violating the inequality $Q[u, v] \geq$ 0 . 


\section{NONLINEAR SYSTEM ASSOCIATED WITH THE OPERATOR $L$}

Let us consider a system of time dependent non-linear equation studied in [9]

$$
L_{t}=[L, M]
$$

Here

$$
M=8 \frac{d^{3}}{d x^{3}}+6 u \frac{d}{d x}+3 u^{\prime}
$$

$u=u(x, t), v=v(x, t)$ and $L_{t} \Psi=\partial u_{t} \partial \Psi+v_{t} \Psi$. Equation (7.1) is equivalent to the following nonlinear system

$$
\begin{gathered}
u_{t}=10 u^{\prime \prime \prime}+6 u u^{\prime}-24 v^{\prime}, \\
v_{t}=3\left(u^{\prime \prime \prime \prime \prime}+u u^{\prime \prime \prime}+u^{\prime} u^{\prime \prime}\right)-8 v^{\prime \prime \prime}-6 u v^{\prime} .
\end{gathered}
$$

Let us consider a 1-soliton solution which corresponds to some negative eigenvalue $k_{*}^{4}, k_{*}=(1+i) s_{*}, s_{*}>0$. Here we give a description of the dynamics of the coefficients $c$ and $d$ introduced in (3.7).

Differentiating the equation $L \Psi_{4}=k^{4} \Psi_{4}$ with respect to $t$ we obtain

$$
\left(L-k^{4}\right)_{t} \Psi_{4}(x, k, t)+\left(L-k^{4}\right)\left(\Psi_{4}(x, k, t)\right)_{t}=0 .
$$

Taking into account (7.1) the latter equation can be rewritten as

$$
M \Psi_{4}(x, k, t)+\left(\Psi_{4}(x, k, t)\right)_{t}=\hat{\Psi}(x, k, t),
$$

for some $\hat{\Psi}(x, k, t)$ such that $\left(L-k^{4}\right) \hat{\Psi}=0$.

Comparing the asymptotics of $\Psi_{4}(x, k, t)$ and the left hand side of (7.2) as $x \rightarrow \pm \infty$ we find from (2.2) and (2.3) that $8 i k^{3} \Psi_{4}(x, k, t)=\hat{\Psi}(x, k, t)$ and then automatically (see (2.8)) $\left(a_{4}\right)_{t}(k, t)=0$. It follows from (3.7) that

$$
\operatorname{res}_{k=k_{*}} \Psi_{4}(x, k, t)=c(t) \Psi_{2}\left(x, k_{*}, t\right)+d(t) \Psi_{3}\left(x, k_{*}, t\right)
$$

and we can rewrite the equation (7.2) as

$$
\begin{gathered}
8\left(-i k_{*}\right)^{3}\left(c(t) \Psi_{2}\left(x, k_{*}, t\right)+d(t) \Psi_{3}\left(x, k_{*}, t\right)\right)= \\
=M\left(c(t) \Psi_{2}\left(x, k_{*}, t\right)+d(t) \Psi_{3}\left(x, k_{*}, t\right)\right) \\
\quad+\left(c(t) \Psi_{2}\left(x, k_{*}, t\right)+d(t) \Psi_{3}\left(x, k_{*}, t\right)\right)_{t} .
\end{gathered}
$$

If we now instead of $\Psi_{2}$ and $\Psi_{3}$ substitute their asymptotics at $+\infty$ (see (2.2)) we obtain

$$
\begin{gathered}
8\left(-i k_{*}\right)^{3} c(t) e^{i k_{*} x}+8\left(-i k_{*}\right)^{3} d(t) e^{-k_{*} x}= \\
=8\left(i k_{*}\right)^{3} c(t) e^{i k_{*} x}+8\left(-k_{*}\right)^{3} d(t) e^{-k_{*} x}+c_{t}(t) e^{i k_{*} x}+d_{t}(t) e^{-k_{*} x}
\end{gathered}
$$

and consequently

$$
c(t)=c(0) \exp \left(-32 s_{*}^{3} t(1+i)\right), \quad d(t)=d(0) \exp \left(-32 s_{*}^{3} t\right) .
$$

This dynamics preserves the conditions of solvability of the system of equations (3.13) $\operatorname{Re} d=0$ and $|d|>|c|$. Therefore a nonsingular soliton remains 
to be nonsingular for all $t$ though its shape might vary. A stationary solution (solution of a constant shape) exists only in the case $c=0$. In this case $u(x, t)=u\left(x+16 s_{*}^{2} t\right)$ and $v(x, t)=v\left(x+16 s_{*}^{2} t\right)$.

Acknowledgements. The authors are grateful to the Institute MittagLeffler for its hospitality during the fall semester 2002. V. Sukhanov and R.Shterenberg are grateful to the Royal Institute of Technology in Stockholm for its hospitality, for financial support from the Gustafsson's foundation and from the Royal Swedish Academy of Science. A support by the RFFI grant 05-01-01076 is also greatly acknowledged. A.Laptev thanks a partial support by the SPECT ESF European programme. J. Östensson would like to thank the Wenner-Gren Foundations for their financial support. All the authors are grateful to J.Hoppe for useful discussions.

\section{REFERENCES}

[1] R. Beals, P. Deift and C. Tomei, Direct and inverse scattering on the line, Mathematical surveys and monographs (AMS) 28 (1988), 1-209.

[2] R. Beals, P. Deift and X. Zhou, The inverse scattering transform on the line. Important developments in soliton theory, Springer Ser. Nonlinear Dynam., Springer, Berlin, (1993), 7-32.

[3] R.D. Benguria, I. Catto, J. Dolbeault and R. Monneau, Oscillating minimizers of a fourth-order problem invariant under scaling, J. Differential Equations 205 (2004), no. 1, 253-269.

[4] P. Deift, Inverse scattering on the line - an overview, Differential equations and mathematical physics (Birmingham, AL, 1990), Math. Sci. Engrg., Academic Press, Boston, MA, 186 (1992), 45-62.

[5] P. Deift and E. Trubowitz, Inverse scattering on the line, Comm. Pure Appl. Math. 32 (1979), no. 2, 121-251.

[6] P. Deift and X. Zhou, Direct and inverse scattering on the line with arbitrary singularities, Comm. Pure Appl. Math. 44 (1991), no. 5, 485-533.

[7] L.D. Faddeev, The inverse problem in the quantum theory of scattering, Uspehi Mat. Nauk (Russian), 14 (1959), no. 4 (88), 57-119.

[8] I.M. Gelfand and L.A.Dikey, Fractional powers of operators, and Hamiltonian systems., Funct. Anal. and Appl. (Russian), 10 (1976), no. 4 (88), 13-29.

[9] J. Hoppe , A. Laptev and J. Östensson, Follytons and the removal of eigenvalues for fourth order differential operators, Preprint math-ph/0311011

[10] V.V. Sukhanov, An inverse problem for a selfadjoint differential operator on the line., Mat. sbornik, (Russian), 137 (2), (1988) 242-259; translation in Math. USSR Sbornik, 65(1), (1990), 249-266.

[11] X. Zhou, Inverse scattering transform for systems with rational spectral dependence, J. Diff. Eq., 115 (1995), 277-303.

[12] X. Zhou, $L^{2}$-Sobolev space bijectivity of the scattering and inverse scattering transforms, Comm. Pure Appl. Math., 51 (1998), 697-731.

[13] J. Östensson, Trace formulae and spectral properties of fourth order differential operators, $\mathrm{KTH}, \mathrm{PhD}$-thesis. 